\title{
Impact of an antibiotic stewardship programme in a surgical setting
}

\section{Authors:}

Muhammad A. Bashar ${ }^{1,2}$ (1)

Jacqui $\mathrm{Miot}^{3}$ (1)

Evan Shoul ${ }^{3}$ (1)

Robyn L. van Zyl ${ }^{1}$ (1)

\section{Affiliations:}

${ }^{1}$ Pharmacology Division,

Department of Pharmacy and Pharmacology, Faculty of Health Sciences, University of the Witwatersrand, Johannesburg, South Africa

${ }^{2}$ Department of

Pharmacology and

Therapeutics, College of

Health Sciences, Federal

University Birnin Kebbi,

Birnin Kebb, Nigeria

${ }^{3}$ Department of Internal Medicine, Faculty of Health Sciences, University of the Witwatersrand,

Johannesburg, South Africa

\section{Corresponding author:}

Muhammad Bashar,

1332258@students.wits.ac. za

\section{Dates:}

Received: 07 June 2021

Accepted: 06 Oct. 2021

Published: 24 Nov. 2021

How to cite this article: Bashar MA, Miot J, Shoul E, Van Zyl RL. Impact of an antibiotic stewardship programme in a surgical setting. S Afr J Infect Dis. 2021;36(1), a307. https://doi. org/10.4102/sajid.v36i1.307

\section{Copyright:}

(c) 2021. The Authors. Licensee: AOSIS. This work is licensed under the Creative Commons Attribution License.

\section{Read online:}

Background: Antibiotics are miracles of science and critical for many surgical procedures. However, the emergence of multidrug resistant pathogens resulting from inappropriate antibiotic use is a threat to modern medicine. This study aimed to determine the appropriateness of antibiotic use, cost, consumption and impact of an antibiotic stewardship intervention round in a surgical ward setting.

Methods: Baseline antibiotic utilisation was determined with a retrospective cross-sectional study in two surgical wards in a tertiary academic hospital in South Africa where medical records of 264 patients who received antibiotics were reviewed. In the second stage of the study, records of 212 patients who received antibiotics were reviewed during a weekly antibiotic stewardship intervention round. The volume of antibiotics consumed was determined using defined daily doses (DDDs)/1000 patients' days, and the appropriateness of the antibiotic prescription for treatment was also determined using a quality-of-use algorithm.

Results: There was a reduction in the volume of antibiotic consumption from a total 739.30 DDDs/1000 to 564.93 DDDs/1000 patient days, with reduction in inappropriate antibiotic use from $35 \%$ to $26 \%$ from baseline to antibiotic stewardship programme stages, respectively. There was an overall increase in culture targeted therapy in both wards in the antibiotic stewardship programme stage.

Conclusion: The implementation of an antibiotic stewardship programme led to a reduction in antibiotic consumption and improvement in appropriate use of antibiotics.

Keywords: antibiotic; antibiotic resistance; antibiotic stewardship; surgery; South Africa; antibiotic prophylaxis; surgical site infection; antibiotic usage.

\section{Introduction}

Antibiotics are a precious resource whose discovery has transformed modern medicine by playing a critical role in the fight against infectious diseases and decreasing mortality caused by bacterial infections. ${ }^{1,2}$ The rapid development of resistance to available antibiotics by bacteria, and lack of development of new agents over the years has negatively affected this initial success. ${ }^{2}$ Antibiotic resistance (ABR) is a global threat to both public health and economic stability, especially in developing countries. A United Kingdom (UK) study predicted that if left unchecked, ABR will claim an extra 10 million lives annually causing the global economy a loss of over US\$ 100 trillion by $2050 .{ }^{3}$ Hence, the urgent need for implementation of successful antibiotic stewardship programmes (ASP) to slow down resistance to antibiotics and conserve last line treatments.

Many surgical conditions like diverticulitis, appendicitis and cholecystitis are infectious in nature, and often treated with antibiotics, ${ }^{4}$ therefore, surgeons need to use antibiotics prudently. Despite advancements in prevention and control of infections, surgical site infections (SSIs) have remained leading cause for mortality and morbidity. ${ }^{5}$ Patients with SSIs are more likely to be readmitted with a higher risk of death, than those without these infections, they also require longer hospitalisation and incur considerable increases in healthcare costs. ${ }^{5}$ Optimising antimicrobial use before, during or after surgical procedures is critical in addressing ABR, simultaneously reducing the burden of infection globally. ${ }^{6}$ This requires a systematic approach by way of antibiotic stewardship to optimise rational antibiotic use. A limited number of studies have been conducted to determine the appropriateness of antibiotic therapy in surgical settings, especially in developing countries. ${ }^{7}$ Hence, the need to involve surgeons in ASPs. An Egyptian study demonstrated that engaging with surgeons with interests in rational antibiotic prescribing to educate their colleagues, can improve optimal antibiotic use for surgical prophylaxis in hospitals. ${ }^{8}$ 
Antimicrobial stewardship has been described as: ${ }^{9}$

[C]oordinated interventions designed to improve and measure the appropriate use of antimicrobial agents by promoting the selection of the optimal antimicrobial drug regimen including dosing, duration of therapy, and route of administration. (p. 323)

An ASP involves several strategies with different approaches, which when properly implemented will improve patient outomes. ${ }^{9}$ The main aim of ASPs are to ensure that patients receive appropriate antibiotic therapy, improve clinical response, with reduced adverse outcomes. ${ }^{10}$ Antibiotic resistance is a direct function of antibiotic use, as the volume of antibiotics consumed increases, so do the chances of developing resistance by bacteria as a survival mechanism. ${ }^{11}$ Over-consumption of antibiotics is a major driver of $\mathrm{ABR}_{1}, 12,13,14$ therefore, appropriate consumption in an ASP helps improve management of antibiotic utilisation. ${ }^{15}$ Apart from reducing the emergence of resistance, ASPs are also associated with reductions in drug acquisition cost, toxicities and infections caused by pathogenic bacteria such as Clostridioides difficile. ${ }^{16}$ To help address the effects of ABR and optimise antibiotic use, the South African Antibiotic Stewardship Programme (SAASP) has published a pocket guide to help prescribers. ${ }^{17}$

A survey to assess the level of implementation of ASP across continents has shown that in Africa only $14 \%$ of hospitals had such programmes in place, compared to $53 \%$ in Asia. ${ }^{18}$ An initial implementation of ASPs in South African public and private hospitals led to a reduction of antibiotic consumption, ${ }^{19,20,21}$ and also resulted in antibiotic cost reduction. ${ }^{19,20}$ However, they were implemented in medical ward settings and did not specifically address ASPs in surgical wards. This study aims to determine the appropriateness of antibiotic use, cost, consumption and impact of an antibiotic stewardship round in a surgical ward setting.

\section{Methods}

\section{Setting}

Charlotte Maxeke Johannesburg Academic Hospital is a tertiary academic teaching hospital of the University of the Witwatersrand, providing a wide range of specialist care and serves as a referral hospital for many hospitals in the Gauteng province and all over Africa. The study was conducted in the vascular (395) and general/gastroenterology (396) surgical wards of this hospital.

\section{Study design}

The study was conducted in two stages as follows.

\section{Baseline stage}

This was a retrospective, cross-sectional medical record review study of patients admitted and who received antibiotics $(n=264)$ in the study wards from February 2016 to May 2016. The variables assessed included: age, gender, diagnosis, indication for surgery, types of antibiotic used, indication, dosage, route of administration, allergy, nature of specimen collected for culture and sensitivity, and biomarker results. These biomarkers included: procalcitonin and C-reactive protein to guide and monitor antibiotic therapy. Results from the National Health Laboratory Services (NHLS) database were used to complement pathology results obtained from patient records. The total volume of antibiotics consumed was determined using defined daily doses (DDDs)/1000 patient days. ${ }^{22,23}$ The number of DDDs for each antibiotic was determined by converting the total amount of that antibiotic used over a given duration into grams and the result was divided by the standard World Health Organization (WHO) DDD value of that antibiotic in grams. The appropriateness of antibiotics used for therapeutic purposes was determined using a guideline developed by Gyssens et al. ${ }^{24}$ The appropriateness of surgical prophylaxis was determined based on the recommendations of the SAASP, ${ }^{17}$ and Essential Medicines Lists for South Africa. ${ }^{25}$ Each prescription was compared to what should be the best practice according to these guidelines and assessed against the Gyssens' categories to determine whether they were appropriate or not (Table 1). The choice of prophylactic agent, dose and duration of prophylaxis was assessed, however the time of the administration of first dose before first surgical incision was not assessed.

\section{Antibiotic stewardship programme stage}

The ASP stage was conducted from June to September 2016, and involved a dedicated ASP weekly round in both study wards. This was separate from the daily routine round conducted by the surgeons. The antibiotic stewardship round was led by an infectious diseases specialist with a team consisting of a pharmacist, a MSc in Clinical Pharmacology from the University Pharmacology Division with the support of a clinical microbiologist. A pre-antibiotic round was conducted by the lead investigator the day before the main round to retrieve results of laboratory investigations and obtain consent from patients to participate in the study. On

TABLE 1: Gyssen's algorithms for evaluation of the appropriateness of antimicrobial drug therapy.

\begin{tabular}{|c|c|}
\hline Category & Reason categorisation \\
\hline 1 & $\begin{array}{l}\text { Agree with the use of antimicrobial therapy/ prophylaxis, the } \\
\text { prescription is definitely appropriate }\end{array}$ \\
\hline II & $\begin{array}{l}\text { The antimicrobial drug therapy/prophylaxis prescription is } \\
\text { inappropriate due to: }\end{array}$ \\
\hline Ila & 1. improper dosage \\
\hline $\mathrm{llb}$ & 2. improper dosage interval \\
\hline IIc & 3. improper route \\
\hline III & $\begin{array}{l}\text { The antimicrobial drug therapy/prophylaxis prescription is } \\
\text { inappropriate due to: }\end{array}$ \\
\hline IIla & 1. excessive length \\
\hline $\mathrm{lllb}$ & 2. duration too short \\
\hline IV & $\begin{array}{l}\text { The antimicrobial drug therapy/prophylaxis prescription is } \\
\text { inappropriate due to: }\end{array}$ \\
\hline IVa & 1. more effective alternative agent $(A a)$ : specify \\
\hline IVb & 2. less toxic Aa: specify \\
\hline IVc & 3. less expensive Aa: specify \\
\hline IVd & 4. less broad spectrum Aa: specify \\
\hline V & $\begin{array}{l}\text { The antimicrobial drug therapy/prophylaxis prescription is } \\
\text { unjustified: use of any antimicrobial is not indicated }\end{array}$ \\
\hline VI & Records insufficient for categorisation. \\
\hline
\end{tabular}

Gyssens IC, Van Den Broek PJ, Kullberg B-J, Hekster YA, Van Der Meer JW. Optimizing antimicrobial therapy. A method for antimicrobial drug use and evaluation. J Antimicrob Chemother. 1992;30(5):724-727. https://doi.org/10.1093/jac/30.5.724 
the day of the ASP ward round, each condition was discussed at the bedside especially regarding antibiotic selection and laboratory investigations with emphasis on collection of appropriate specimens for cultures. Fellows, residents and interns in surgery also participated in the rounds. The ASP interventions also involved early conversion from intravenous to oral agents, dose optimisation and adjustment of dose in patients with renal and hepatic impairment. Patients who were on antibiotics were all reviewed during the rounds, however only consented patients' records were included in the data analysis. All enrolled patients were followed for the duration of their admission stay to ensure compliance of the recommendations made during antibiotic rounds. The same types of variables were collected as in the baseline stage. The appropriateness of antibiotic prescriptions, cost and volume of antibiotic consumed were determined using the same criteria as in the first stage.

\section{Ethical considerations}

The study was approved by the University of the Witwatersrand Human Research Ethics Committee with an approval number: M151142. All participants gave their informed consent for inclusion before they participated in the study.

\section{Statistical analysis}

Data was analysed using Stata software version 14 (StataCorp, College Station, TX, USA). An independent sample $t$-test was used to compare the differences in volume of antibiotic consumption between the two stages. There were 17 and 12 patient records in the baseline and ASP stages, respectively, that were incomplete. The results were mostly related to culture and biomarkers, and they were excluded in the analysis.

\section{Results}

The results of each stage are presented but not compared because of differences in patient populations between the two stages. There were more male patients in both stages of the study as shown in Table 2. The baseline stage of the patients in the baseline stage was $51.50 \pm 15.91$ years, while in the ASP stage it was $45.77 \pm 16.81$ years $(p=0.01)$. The average length of hospital stays (LOS) was significantly increased in the ASP stage $(p=0.01)$. In the baseline stage, amoxicillin/clavulanic acid was the most frequently used antibiotic (53\%), followed by piperacillin/tazobactam $(16 \%)$; while in the ASP stage, although still the most used agent, the frequency of amoxicillin/clavulanic reduced to $34 \%$, while piperacillin/tazobactam increased to $26 \%$ (Table 2).

More specimens were collected for culture in the ASP stage. A total of 397 and 491 cultures were requested in the baseline and ASP stages, respectively. The collection of appropriate specimens such as tissue instead of superficial swab was emphasised in the rounds. The collection of tissue specimens for culture was more in the ASP stage (28.8\%) compared to the baseline stage (15.3\%). The request of superficial swabs for culture in the vascular ward reduced from $22.7 \%$ in the baseline stage to $11.2 \%$ in the ASP stage.

The intravenous route of administration was the most utilised route in both stages, where $89 \%$ and $84 \%$ of all drugs were administered intravenously in the baseline stage and the ASP stage, respectively. There was a higher number of culture targeted therapies prescribed in both wards in the ASP stage compared to the baseline stage (see Appendix 1). Macrolides such as azithromycin were used as prokinetic agents to stimulate gastrointestinal motility in newly operated patients. There was a reduction in the use of antibiotics for empirical therapy in the general ward during the ASP stage.

In the baseline stage, 443 antibiotic prescriptions were ordered for therapeutic purposes, of which 35\% were inappropriate based on the algorithm used, whilst in the ASP stage, 442 antibiotic prescriptions were ordered of which $26 \%$ were inappropriate. In both stages, the main reasons for inappropriate prescriptions were the use of more broadspectrum (Gyssens' category IVd) and more expensive agents (Gyssens' category IVc), where narrow spectrum and less expensive agents were available. Overall, 64\% and 59\% of the antibiotic use for prophylactic indications in the baseline and ASP stages, respectively, was inappropriate (Table 3). Amoxicillin/clavulanic acid was the most commonly used agent (68\%) for surgical prophylaxis in the baseline stage followed by cefazolin (29\%). According to the classification system used, based on the South African guidelines, nearly $65 \%$ of all surgical prophylaxis was inappropriate based on the agent choice, while $7.3 \%$ was inappropriate based on duration of prophylaxis. Similarly, in the ASP stage, amoxicillin/clavulanic acid and cefazolin were the two most frequently used agents for surgical prophylaxis, and $62 \%$ of all surgical prophylaxis in this stage was inappropriate based on choice of agent; with $6.6 \%$

TABLE 2: Patient demographics and clinical indicators.

\begin{tabular}{|c|c|c|c|}
\hline Variables & Baseline stage (\%) & ASP stage (\%) & $p$ \\
\hline Demographics & - & - & - \\
\hline Number of patients & 264 & 212 & - \\
\hline Gender & & & 0.501 \\
\hline Male & $150(56.82)$ & $114(53.77)$ & - \\
\hline Female & $114(43.18)$ & $98(46.23)$ & - \\
\hline Age (years) Mean + s.d. & $45.77 \pm 16.81$ & $51.50 \pm 15.91$ & 0.01 \\
\hline LOS (days) & $8.33 \pm 7.58$ & $13.44 \pm 11.83$ & 0.01 \\
\hline $\begin{array}{l}\text { DoT for therapeutic } \\
\text { purposes (days) }\end{array}$ & $4.74 \pm 4.58$ & $3.96 \pm 2.04$ & 0.01 \\
\hline $\begin{array}{l}\text { Antibiotic consumption } \\
\text { (DDD/1000 patient days) }\end{array}$ & 739.30 & 564.93 & 0.038 \\
\hline $\begin{array}{l}\text { Total number of antibiotic } \\
\text { prescriptions (percentage } \\
\text { of inappropriate antibiotic } \\
\text { used therapeutically) }\end{array}$ & $443(35)$ & $442(26)$ & 0.006 \\
\hline $\begin{array}{l}\text { Total prescriptions } \\
\text { administered IV }\end{array}$ & $396(89.39)$ & $372(84.16)$ & 0.341 \\
\hline $\begin{array}{l}\text { Appropriately prescribed IV } \\
\text { antibiotics }\end{array}$ & $252(56.90)$ & $269(60.80)$ & 0.302 \\
\hline
\end{tabular}

ASP, antibiotic stewardship programmes; s.d., standard deviation, LOS, length of hospital stay; DoT, duration of antibiotic therapy; DDDs, defined daily doses; IV, intravenous. $p$ values calculated using the chi-square test or $t$-test where appropriate. 
TABLE 3: Appropriateness of peri-operative antibiotic prophylaxis.

\begin{tabular}{|c|c|c|}
\hline Variables & Baseline stage (\%) & ASP stage $(\%)$ \\
\hline Overall inappropriate use & 64 & 59 \\
\hline Inappropriateness due to agent choice & 65 & 62 \\
\hline $\begin{array}{l}\text { Inappropriateness due to duration } \\
\text { more than } 24 \mathrm{~h}\end{array}$ & 7.3 & 6.6 \\
\hline $\begin{array}{l}\text { Time of the administration of the first } \\
\text { dose }\end{array}$ & Not assessed & Not assessed \\
\hline
\end{tabular}

ASP, antibiotic stewardship programmes.

inappropriate based on the duration of prophylaxis. Piperacillin-tazobactam was given at $4.5 \mathrm{~g}$ qid while cefazolin was administered at the dose of either $1 \mathrm{~g}$ or $2 \mathrm{~g}$ tds. in both wards. Up to $71 \%$ of the recommendations made during the antibiotic stewardship rounds were implemented. Reasons were not given on why some of the recommendations were not implemented.

A reduction (24\%) in the total volume of antibiotic consumption was observed after implementation of the antibiotic stewardship round by 174.37 DDD/1000 patient days. The trend of the total monthly antibiotic consumption in the two stages is shown in Figure 1. There was an increase in the utilisation of piperacillin/tazobactam in the ASP stage (Table 4), which was because of an increase in the prevalence of Pseudomonas aeruginosa compared to the baseline stage (Appendix 2). Consumption of the five most commonly used agents in both stages of the study is presented based on clinical indications in Appendix 3.

\section{Discussions}

This ASP intervention study was conducted in a surgery setting of a South African academic hospital with an antibiotic stewardship team led by an infectious diseases specialist. Overconsumption and inappropriate use of antibiotics are major drivers of $\mathrm{ABR}$ with consequent additional hospital costs. ${ }^{11}$ Inappropriate use of antibiotics includes use of antibiotics at suboptimal doses, incorrect route of administration or poor adherence to prescribed drugs. ${ }^{26}$ It also involves the use of antibiotics without a defined bacterial/fungal infection or an indication for prophylaxis, ${ }^{26}$ and the use of last resort agents, while there are effective first and second line antibiotics. ${ }^{27}$

A baseline stage was used to describe the current standard of antibiotic usage in each of the wards. Both the baseline and ASP ward round targeted all patients on antibiotics. A wide range of surgical cases were admitted in the general ward. The cases included obstructed hernias, intestinal obstruction and cutaneous abscesses, perforated peptic ulcer diseases, and oesophageal carcinomas. Others are: lower gastrointestinal bleeding, rectal carcinomas, acute cholangitis, cholecystitis, pyogenic liver abscesses, primary liver cell carcinomas, peri-pancreatic sepsis, appendicitis, and overwhelming post-splenectomy sepsis. In the vascular ward, the cases included acute and chronic vascular ischemic diseases, diabetic foot diseases, stab injuries, and many other vascular conditions.

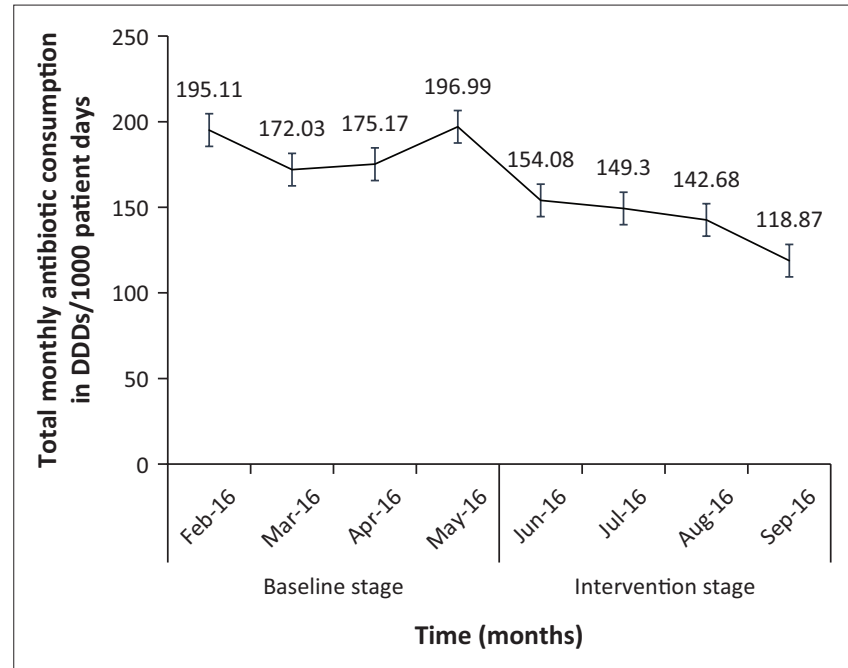

DDDs, defined daily doses.

FIGURE 1: Trend of antibiotic consumption over the study period.

TABLE 4: Frequency of top 10 antibiotic prescribed over a 10 -month period in the general and vascular surgical wards.

\begin{tabular}{|c|c|c|c|c|c|c|}
\hline \multirow[t]{2}{*}{ Name of antibiotics } & \multicolumn{2}{|c|}{$\begin{array}{c}\text { Number of } \\
\text { prescriptions (\%) } \\
\text { at baseline stage }\end{array}$} & \multicolumn{2}{|c|}{$\begin{array}{l}\text { Number of } \\
\text { prescriptions (\%) } \\
\text { at ASP stage }\end{array}$} & \multicolumn{2}{|c|}{$\begin{array}{c}\text { Difference in } \\
\text { number }(\%) \text { of } \\
\text { prescriptions issued }\end{array}$} \\
\hline & $n$ & $\%$ & $n$ & $\%$ & $n$ & $\%$ \\
\hline $\begin{array}{l}\text { Amoxicillin/ } \\
\text { clavulanic acid }\end{array}$ & 233 & 52.59 & 149 & 33.71 & $\uparrow 84$ & 18.88 \\
\hline $\begin{array}{l}\text { Piperacillin/ } \\
\text { tazobactam }\end{array}$ & 72 & 16.26 & 115 & 26.02 & $\downarrow-43$ & -9.76 \\
\hline Cefazolin & 36 & 8.13 & 31 & 7.21 & $\uparrow 5$ & 0.92 \\
\hline Metronidazole & 20 & 4.52 & 18 & 4.07 & $\uparrow 2$ & 0.45 \\
\hline Fluconazole & 13 & 2.93 & 19 & 4.29 & $\downarrow-6$ & -1.36 \\
\hline Vancomycin & 10 & 2.26 & 6 & 1.39 & $\uparrow 4$ & 0.87 \\
\hline Cloxacillin & 7 & 1.58 & 3 & 0.68 & 个 4 & 0.90 \\
\hline Imipenem & 7 & 1.58 & 8 & 1.81 & $\downarrow-1$ & -0.23 \\
\hline Ciprofloxacin & 6 & 1.35 & 13 & 2.72 & $\downarrow-7$ & -1.37 \\
\hline Ertapenem & 2 & 0.45 & 18 & 4.07 & $\downarrow-16$ & -3.62 \\
\hline Others & 37 & 8.35 & 62 & 14.03 & $\downarrow-25$ & -5.68 \\
\hline Total & 443 & 100.00 & 442 & 100.00 & - & - \\
\hline
\end{tabular}

ASP, antibiotic stewardship programmes.

The demographic characteristics of the patients show that more male patients were admitted to the surgical wards in both stages of the study, which is mirrored in other studies conducted in other countries. ${ }^{28,29}$ The patients in the ASP stage had a higher mean age compared to those in the baseline stage which may have clinical implications in that older patients may have a higher risk of having other comorbidities, complications or severity of disease. Previous studies in surgical settings conducted in Malaysia and Jordan reported average ages of between 43 and 51 years, ${ }^{6,30}$ respectively, which is similar to those reported in this study. Although many ASPs have reported a reduction in LOS during interventions, ${ }^{22,31}$ in contrast, this study showed an increase in LOS. The average LOS of patients in the baseline and ASP stages were $8.33 \pm 7.58$ and $13.44 \pm 11.83$ days, respectively. This may be because of the fact that this study was conducted in a surgical setting and older patients were admitted during the ASP stage compared to baseline stage. Another reason for an increased LOS in the ASP stage was an increase in the prevalence of Pseudomonas aeruginosa in the 
ASP stage, which was statistically significant $(p=0.01)$ compared to the baseline stage. Despite an increase in LOS in this study, there was a reduction in antibiotic consumption and duration of antibiotic therapy (DoT) in the ASP stage. During ASP rounds, it was found that many patients (especially those with ischemic vascular conditions) stayed longer on admission waiting for surgical procedures. As most of them did not have infections, it was unnecessary for them to be on antibiotics so these were stopped during ward rounds. The number of laboratory tests per patient may be driven by an increased length of stay where more tests are ordered with a longer LOS.

Globally there is no standard metric for quantifying antibiotic consumption across all clinical settings, ${ }^{32}$ and the WHO has recommended the use of DDDs, ${ }^{18}$ defined as 'the average maintenance dose of the drug when used for its primary indication in adults' ${ }^{33}$ Expressing antibiotic consumption by DDDs/1000 patient days enables comparison between hospitals ${ }^{33}$ and clinical settings, as was implemented in this study. A key finding in this study was lower antibiotic consumption in the ASP stage. The ASP programmes in South African public and private hospitals have also reported reductions in antibiotic consumption, ${ }^{19,20,21}$ as well as in developed countries. . $^{234,35}$

The use of antibiotics when there is no indication for their use, such as to treat clean wounds and using it for prolonged duration or failure to de-escalate to narrow spectrum when the results of culture are available, are considered inappropriate. To encourage appropriate antibiotic use it was emphasised during rounds that antibiotics should only be used empirically when there is clear indication, and effort should be made to collect appropriate specimens for culture before starting empiric treatment and should be reviewed when the culture results are available. There were discussions during the rounds on what is considered appropriate such as prescribing antibiotics at the right dose via the right route of delivery. It was previously reported that both public and private Intensive Care Units (ICUs) across South Africa had a high percentage of inappropriate use of antibiotics of $44 \%$ and $61 \%$ in public and private ICUs, respectively. ${ }^{36}$ This is higher than what is observed in the current study $(26 \%-35 \%)$, possibly because those studies were conducted in the ICU setting where higher antibiotic consumption is more likely. Studies in other developing countries such as India and Malaysia have also shown a high percentage $(39 \%-42 \%)$ of inappropriate antibiotic usage. ${ }^{7,29}$ However, this is not limited to developing countries, a Swiss study using Gyssens' algorithm found a total of $32 \%$ of all prescriptions to be inappropriate which was high $(37 \%)$ in patients who received antibiotics as treatment compared to those who received it for prophylaxis $(17 \%) \cdot{ }^{37} \mathrm{In}$ the Netherlands, $16 \%-29 \%$ of all antibiotic prescriptions were judged inappropriate using Gyssens' algorithm, and this was mostly because of unjustified prescriptions..$^{38,39}$

Surgical prophylaxis is an area of concern with respect to appropriate antibiotic usage. A high proportion of inappropriate antibiotic utilisation was seen in prophylactic usage in the baseline (65\%) and ASP (62\%) stages of this study, based on the wrong choice of agents, while $7.3 \%$ and $6.6 \%$ were inappropriate based on a duration of more than the recommended $24 \mathrm{~h}$, respectively. Of the antibiotics prescribed, amoxicillin/clavulanic acid was the most frequently used prophylactic agent in both stages. The use of amoxicillin/clavulanic acid is not recommended for surgical prophylaxis by the SAASP and Standard Treatment Guidelines and Essential Medicines Lists for South Africa for most of the procedures conducted on patients at the time of this study. ${ }^{17,25}$ Augmentin and cefazolin are prescribed prophylactically in theatre at doses of $1.2 \mathrm{~g}$ bd and $1 \mathrm{~g}$ tds, respectively, but are then often continued unnecessarily for days on the wards.

Inappropriate use of prophylaxis has been found to be even higher in surgical settings of other developing countries; a Malaysian study found $66 \%$ of prophylaxis to be inappropriate, with $35 \%$ because of inappropriate duration and $16 \%$ as a result of wrong choice. ${ }^{7}$ A study in a Jordanian cardiac surgery centre, found that up to $98 \%$ of surgical prophylaxis was inappropriate based on wrong antibiotic choice, while 59\% was inappropriate with a duration of more than $48 \mathrm{hr}$ as recommended by the guideline. ${ }^{30} \mathrm{~A}$ study in a private surgical setting in India found that $32 \%$ of surgical prophylaxis was inappropriate because of choice of agents, while 37\% was inappropriate on account of prolonged duration of prophylaxis. ${ }^{40}$ Appropriate surgical prophylaxis reduces the risk of potential contamination during surgery, thus lowering the chance of SSIs. ${ }^{41}$ On the other hand, inappropriate surgical prophylaxis is associated with drug-related adverse events and Clostridioides difficile infections at the individual level, and increases the risk of developing resistant pathogens and healthcare costs at the community level..$^{42}$

A lower mean DoT for therapeutic purposes was found in the ASP stage of $3.96 \pm 2.04$ days compared to $4.74 \pm 4.58$ days in the baseline stage. A short DoT with an antibiotic is recommended by the World Society of Emergency Surgery in the management of intra-abdominal infections. ${ }^{43}$ A short DoT reduces the risk of developing resistance, adverse events and cost, and is recommended in all patients except in situations where there is difficulty in achieving source control, sepsis, and in immunocompromised individuals.

The programme was well accepted in the wards with no resistance from the surgeons or ward staff. During the programme, a $71 \%$ compliance rate to the recommendations was recorded and although this is a favourable outcome considering the short duration of the study, it is lower than that reported in other studies. ${ }^{44,45}$ The compliance rate may have been better if surgical consultants participated more actively in the rounds. In this study, it was found that Gyssens' algorithm is a useful and reliable tool in determining appropriateness of antibiotic prescriptions and can be recommended to be used in future studies. In the authors' opinion, the incorporation of antibiotic stewardship rotations and inclusion of short courses on appropriate 
antibiotic use in the management of surgical infections in the curriculum of South African surgery-related residency programmes will go a long way in ensuring optimal rational antibiotic usage and reducing the burden of SSIs. These results have clinical implications in that the level of inappropriate antibiotic usage for surgical prophylaxis in baseline and ASP is high, mostly because of incorrect agents being prescribed and for a prolonged duration of prophylaxis. This may increase rates of resistance, chances of acquiring SSIs and additional costs to the patients. The high rate of inappropriate surgical prophylaxis in the ASP stage was most likely on account of the majority of antibiotics being started in the operating theatre, whereas this intervention was conducted in the wards. Possible measures to improve appropriate antibiotic use for surgical prophylaxis could include adherence to national guidelines and educational programmes targeting theatre staff and surgeons on rational antibiotic usage.

\section{Study limitations}

A limitation of this study is that it was conducted at two different time points and therefore it was not possible to determine if there was a significant impact of the ASP ward rounds on inappropriate antibiotic usage. Another limitation is that it was conducted in only two surgical wards of an academic hospital and this makes it difficult to generalise the findings. It may be problematic to replicate this study in many non-teaching hospitals in South Africa and across the African continent, because of the shortage of ASP experts outside of the academic setting. The Netcare model in which non-specialist pharmacists successfully drove the ASP in a South African group of private hospitals is an alternative option. ${ }^{21}$ Although the ASP ward round in this study showed a lower volume of antibiotic consumption and inappropriate antibiotic use compared to the baseline stage, which are important drivers of resistance, it was not possible to say this would lead to a reduction in ABR, because the study was conducted over a short period of time. Data collection was challenging because of lack of electronic patient records as well as monitoring the implementation of recommendations made during the antibiotic stewardship rounds.

\section{Conclusion}

Introduction of a weekly ASP round in two surgical wards of a tertiary South African hospital showed a lower level of antibiotic consumption, improvement in the appropriate use of antibiotics and a reduction in the duration of antibiotic therapy. Hospitals in developing countries are encouraged to optimise the use of antibiotics by implementing ASP programmes.

\section{Acknowledgements}

The authors acknowledge the invaluable contributions of all the staff of wards 395 (Vascular) and 396 (General/ gastroenterology), Microbiology Record Room, and the Department of Health Information Record Room of the
Charlotte Maxeke Johannesburg Academic Hospital, Johannesburg for their support during the study. The authors appreciate the funding from the University of the Witwatersrand Faculty of Health Sciences Research Office, Federal University Birnin Kebbi and Tertiary Education Trust Fund (TETFUND), Nigeria for funding B.M.A. MSc studies.

\section{Competing interests}

The authors declare that they have no financial or personal relationships that may have inappropriately influenced them in writing this article.

\section{Authors' contributions}

M.A. is a masters' student and was involved in every step of the study, from study design, data collection, data analysis and writing of the manuscript. J.M., E.S. and R.V.Z., supervised and guided M.A. throughout his MSc programme from study design to data analysis and manuscript writing. All authors approved the final manuscript.

\section{Funding information}

The authors received no financial support for the research, authorship, and/or publication of this article.

\section{Data availability}

The data that support the findings of this study are available from the corresponding author, M.A., upon reasonable request.

\section{Disclaimer}

The views expressed in this manuscript are those of the authors and not an official position of the University of the Witwatersrand.

\section{References}

1. Anand N, Nayak IN, Advaitha M, et al. Antimicrobial agents' utilization and cost pattern in an Intensive Care Unit of a Teaching Hospital in South India. Indian J Crit Care Med. 2016;20(5):274-279. https://doi.org/10.4103/0972-5229.182200

2. Barlam TF, Cosgrove SE, Abbo LM, et al. Implementing an Antibiotic Stewardship Program: Guidelines by the Infectious Diseases Society of America and the Society for Healthcare Epidemiology of America. Clin Infect Dis. 2016;62(10):e51-e77. https://doi.org/10.1093/cid/ciw118

3. O'Neill J. Tackling drug-resistant infections globally: Final report and recommendations [homepage on the Internet]. London: Wellcome Trust \& He Majesty's Government. 2016 [cited 2017 Jan 12]. Available from: https:// amrreview.org/sites/default/files/160518_Final\%20paper_with\%20cover.pdf

4. Leeds IL, Fabrizio A, Cosgrove SE, Wick EC. Treating wisely: The surgeon's role in antibiotic stewardship. Ann Surg. 2017;265(5):871-873. https://doi.org/10.1097/ SLA.0000000000002034

5. Anderson DJ, Podgorny K, Berríos-Torres SI, et al. Strategies to prevent surgical site infections in acute care hospitals: 2014 update. Infect Control Hosp Epidemiol. 2014;35(S2):S66-S88. https://doi.org/10.1086/676022

6. Charani E, Ahmad R, Tarrant C, et al. Opportunities for system level improvement in antibiotic use across the surgical pathway. Int J Infect Dis. 2017;60:29-34. https://doi.org/10.1016/j.ijid.2017.04.020

7. Lim MK, Lai PSM, Ponnampalavanar SSLS, et al. Antibiotics in surgical wards: use or misuse? A newly industrialized country's perspective. J Infect Dev Ctries. 2015;9(11):1264-1271. https://doi.org/10.3855/jidc.6731

8. Saied T, Hafez SF, Kandeel A, et al. Antimicrobial stewardship to optimize the use of antimicrobials for surgical prophylaxis in Egypt: A multicenter pilot intervention study. Am J Infect Control. 2015;43(11):e67-e71. https://doi.org/10.1016/j. ajic.2015.07.004 
9. Society for Healthcare Epidemiology of America; Infectious Diseases Society of America; Pediatric Infectious Diseases Society. Policy statement on antimicrobial America, Pediatric stewardship by the Society for Healthcare Epidemiology of America (SHEA), the Infectious Diseases Society of America (IDSA), and the Pediatric Infectious Diseases Society (PIDS). Infect
https://doi.org/10.1086/665010

10. Davey $P$, Brown $E$, Charani $E$, et al. Interventions to improve antibiotic prescribing practices for hospital inpatients. Cochrane Database Syst Rev. 2013. Art. No. CD003543. https://doi.org/10.1093/jac/dkm154

11. Gelband H, Miller P, Molly PS, et al. Centre for Disease Dynamics, Economics \& Policy [homepage on the Internet]. 2015 [cited 2015 Dec 02]. Available from: https://cddep.org/sites/default/files/swa_2015_final.pdf

12. Dellit TH, Owens RC, Mcgowan JE, et al. Infectious Diseases Society of America and the Society for Healthcare Epidemiology of America guidelines for developing an institutional program to enhance antimicrobial stewardship. Clin Infect Dis. 2007;44(2):159-177. https://doi.org/10.1086/510393

13. Lepper PM, Grusa E, Reichl H, Högel J, Trautmann M. Consumption of imipenem correlates with betalactam resistance in Pseudomonas aeruginosa. Antimicrob
Agents Chemother. 2002;46(9):2920-2925. https://doi.org/10.1128/ Agents Chemother.

14. Mendelson $M$, Røttingen J-A, Gopinathan $U$, et al. Maximising access to achieve appropriate human antimicrobial use in low-income and middle-income countries. Lancet. 2016;387(10014):188-198. https://doi.org/10.1016/S01406736(15)00547-4

15. Berrington A. Antimicrobial prescribing in hospitals: Be careful what you measure J Antimicrob Chemother. 2010;65(1):163-168. https://doi.org/10.1093/jac/ dkp399

16. Gould I. Antibiotic resistance: The perfect storm. Int J Antimicrob Ag. 2009;34(Suppl 3):S2-S5. https://doi.org/10.1016/S0924-8579(09)70549-7

17. Wasserman S, Boyles T, Mendelson M. A pocket guide to antibiotic prescribing for adults in South Africa [homepage on the Internet]. Cape Town: Antibiotic
Stewardship Programme. 2015 [cited 2016 Mar 02]. Available from: http://www. bestcare.org.za/file/view/SAASP+Antibiotic+Guidelines_2015.pdf

18. Howard P, Pulcini C, Hara GL, et al. An international cross-sectional survey of antimicrobial stewardship programmes in hospitals. J Antimicrob Chemother. 2015;70(4):1245-1255. https://doi.org/10.1093/jac/dku497

19. Boyles TH, Whitelaw A, Bamford C, et al. Antibiotic stewardship ward rounds and a dedicated prescription chart reduce antibiotic consumption and pharmacy costs without affecting inpatient mortality or re-admission rates. PLoS One. 2013;8(12):e79747. https://doi.org/10.1371/journal.pone.0079747

20. Boyles T, Naicker V, Rawoot N, Raubenheimer P, Eick B, Mendelson M. Sustained reduction in antibiotic consumption in a South African public sector hospital; Fou year outcomes from the Groote Schuur Hospital antibiotic stewardship program. S Afr Med J. 2017;107(2):115-118. https://doi.org/10.7196/SAMJ.2017.v107i2.12067

21. Brink AJ, Messina AP, Feldman C, et al. Antimicrobial stewardship across 47 South African hospitals: An implementation study. Lancet Infect Dis. 2016;16(9):10171025. https://doi.org/10.1016/S1473-3099(16)30012-3

22. Sintchenko V, Iredell JR, Gilbert GL, Coiera E. Handheld computer based decision support reduces patient length of stay and antibiotic prescribing in critical care. J Am Med Inform Assoc. 2005;12(4):398-402. https://doi.org/10.1197/jamia.M1798

23. Taggart LR, Leung E, Muller MP, Matukas LM, Daneman N. Differential outcome of an antimicrobial stewardship audit and feedback program in two intensive care units: A controlled interrupted time series study. BMC Infect Dis. 2015;15:480491. https://doi.org/10.1186/s12879-015-1223-2

24. Gyssens IC, Van Den Broek PJ, Kullberg B-J, Hekster YA, Van Der Meer JW. Optimizing antimicrobial therapy. A method for antimicrobial drug use and evaluation. Antimicrob Chemother. 1992;30(5):724-727. https://doi.org/10.1093/jac/30.5.724

25. NDoH. National Department of Health South Africa. Standard treatment guidelines and essential medicines lists for South Africa [homepage on the Internet]. 2015 [cited 2016 Sep 20]. Available from: http://www.health.gov.za

26. Starrels JL, Barg FK, Metlay JP. Patterns and determinants of inappropriate antibiotic use in injection drug users. J Gen Intern Med. 2009;24:263-269. https:// antibiotic use in injection drug users.
doi.org/10.1007/s11606-008-0859-7

27. Rottier WC, Bamberg YR, Dorigo-Zetsma JW, Van Der Linden PD, Ammerlaan HS. Bonten MJ. The predictive value of prior colonization and antibiotic use for 3rd generation cephalosporin resistant Enterobacteriaceae bacteremia in patients with sepsis. Clin Infect Dis. 2015;60(11):1622-1630. https://doi.org/10.1093/cid/civ121
28. Zygourakis CC, Lee J, Barba J, Lobo E, Lawton MT. Performing concurrent operations in academic vascular neurosurgery does not affect patient outcomes. J Neurosurg. 2017;127(5):1089-1095. https://doi.org/10.3171/2016.6.JNS16822

29. James E, Venu RP. Appropriateness of antibiotic usage for gastrointestinal disorders in a tertiary care hospital. Eur J Hosp. 2016;23(5):283-287. https://doi. org/10.1136/ejhpharm-2015-000818

30. Al-Momany NH, Al-Bakri AG, Makahleh ZM, Wazaify MM. Adherence to international antimicrobial prophylaxis guidelines in cardiac surgery: A Jordanian study demonstrates need for quality improvement. J Manag Care Spec Pharm. 2009;15(3):262-271. https://doi.org/10.18553/jmcp.2009.15.3.262

31. Nault V, Pepin J, Beaudoin M, Perron J, Moutquin JM, Valiquette L. Sustained impact of a computer-assisted antimicrobial stewardship intervention on antimicrobial use and length of stay. J Antimicrob Chemother. 2016;72(3):933940. https://doi.org/10.1093/jac/dkw468

32. Polk RE, Fox C, Mahoney A, Letcavage J, Macdougall C. Measurement of adult antibacterial drug use in 130 US hospitals: Comparison of defined daily dose and days of therapy. Clin Infect Dis. 2007;44(5):664-670. https://doi. days of therapy.

33. WHO Collaborating Centre for Drug Statistics Methodology. ATC/DDD Index 2016 [homepage on the Internet]. [cited 2016 Mar 03]. Available from: http://www. whocc.no/atc_ddd_index/

34. Cisneros J, Neth O, Gil-Navarro MV, et al. Global impact of an educational antimicrobial stewardship programme on prescribing practice in a tertiary hospital centre. Clin Microbiol Infect. 2014;20(1):82-88. https://doi. org/10.1111/1469-0691.12191

35. Peto Z, Benko R, Matuz M, Csullog E, Molnar A, Hajdu E. Results of a local antibiotic management program on antibiotic use in a tertiary intensive care unit in Hungary. Infection. 2008;36:560-564. https://doi.org/10.1007/s15010-008-7377-8

36. Paruk F, Richards G, Scribante J, Bhagwanjee S, Mer M, Perrie H. Antibiotic prescription practices and their relationship to outcome in South African intensive care units: Findings of the Prevalence of Infection in South African Intensive Care Care units: Findings of the Prevalence of Infection in South African Intensive Care Units (PISA)
SAMJ.5833

37. Cusini A, Rampini SK, Bansal $V$ et al. Different patterns of inappropriate antimicrobial use in surgical and medical units at a tertiary care hospital in Switzerland: A prevalence survey. PLoS One. 2010;5(11):e14011. https://doi. Switzerland: A prevalence survey.
org/10.1371/journal.pone.0014011

38. Akhloufi $H$, Streefkerk $R$, Melles $D$, et al. Point prevalence of appropriate antimicrobial therapy in a Dutch university hospital. Eur J Clin Microbiol Infect Dis. 2015;34:1631-1637. https://doi.org/10.1007/s10096-015-2398-6

39. Willemsen I, Van Der Kooij T, Van Benthem B, Wille J, Kluytmans J. Appropriateness of antimicrobial therapy: A multicentre prevalence survey in the Netherlands, 2008-2009. Euro Surveill. 2010;15(46):pii=19715. https://doi.org/10.2807/ ese.15.46.19715-en

40. Parulekar L, Soman R, Singhal T, Rodrigues C, Dastur F, Mehta A. How good is compliance with surgical antibiotic prophylaxis guidelines in a tertiary care private hospital in India? A prospective study. Indian J Surg. 2009;71:15-18. https://doi org/10.1007/s12262-009-0004-9

41. Bratzler DW, Dellinger EP, Olsen KM, et al. Clinical practice guidelines for antimicrobial prophylaxis in surgery. Am J Health-Syst Pharm. 2013;70(3):195283. https://doi.org/10.2146/ajhp120568

42. Owens RC, Donskey CJ, Gaynes RP, Loo VG, Muto CA. Antimicrobial-associated risk factors for Clostridium difficile infection. Clin Infect Dis. 2008;46(Suppl 1):S19-S31. https://doi.org/10.1086/521859

43. Sartelli $M$, Viale $P$, Catena $F$, et al. WSES guidelines for management of intraabdominal infections. World J Emerg Surg. 2013;8:3-32. https://doi. org/10.1186/1749-7922-8-3

44. Bhalla N, Hussein N, Atari M, et al. Introducing an antibiotic stewardship program in a humanitarian surgical hospital. Am J Infect Control. 2016,44(11):1381-1384. https://doi.org/10.1016/j.ajic.2016.03.036

45. Del Arco A, Tortajada B, De La Torre J, et al. The impact of an antimicrobial stewardship programme on the use of antimicrobials and the evolution of drug
resistance. Eur J Clin Microbiol Infect Dis. 2015;34:247-251. https://doi. org/10.1007/s10096-014-2225-5 


\section{Appendix 1}

TABLE 1-A1: Antibiotic therapy based on clinical indications.

\begin{tabular}{|c|c|c|c|c|c|c|c|c|c|c|c|c|}
\hline \multirow[t]{3}{*}{ Clinical indications } & \multicolumn{6}{|c|}{ Baseline stage number of prescription (\%) } & \multicolumn{6}{|c|}{ ASP stage number of prescription (\%) } \\
\hline & \multicolumn{2}{|c|}{ Vascular ward } & \multicolumn{2}{|c|}{ General ward } & \multicolumn{2}{|c|}{ Total of both wards } & \multicolumn{2}{|c|}{ Vascular ward } & \multicolumn{2}{|c|}{ General ward } & \multicolumn{2}{|c|}{ Total of both wards } \\
\hline & $n$ & $\%$ & $n$ & $\%$ & $n$ & $\%$ & $n$ & $\%$ & $n$ & $\%$ & $n$ & $\%$ \\
\hline Empiric & 58 & 56.31 & 186 & 54.71 & 244 & 55.08 & 82 & 56.94 & 158 & 53.02 & 240 & 54.30 \\
\hline Prophylaxis & 32 & 31.07 & 95 & 27.94 & 127 & 28.67 & 37 & 25.69 & 60 & 20.13 & 97 & 21.95 \\
\hline Targeted & 12 & 11.65 & 55 & 16.18 & 67 & 15.12 & 25 & 17.37 & 63 & 21.15 & 88 & 19.90 \\
\hline Prokinetic & 1 & 0.97 & 4 & 1.17 & 5 & 1.13 & 0 & 0.00 & 17 & 5.70 & 17 & 3.85 \\
\hline Total & 103 & 100.00 & 340 & 100.00 & 443 & 100.00 & 144 & 100.00 & 298 & 100.00 & 442 & 100.00 \\
\hline
\end{tabular}

ASP, antibiotic stewardship programmes. 


\section{Appendix 2}

TABLE 1-A2: Pathogens isolated in the two stages of the study.

\begin{tabular}{|c|c|c|c|}
\hline Cultured pathogens & Baseline stage & ASP stage & $p$ \\
\hline Escherichia coli & 21 & 22 & 0.46 \\
\hline Pseudomonas aeruginosa & 18 & 29 & 0.01 \\
\hline Klebsiella pneumoniae & 17 & 23 & 0.27 \\
\hline Methicillin-sensitive Staphylococcus aureus & 12 & 5 & 0.001 \\
\hline Enterococcus cloacae & 1 & 11 & 0.001 \\
\hline Proteus mirabilis & 11 & 5 & 0.01 \\
\hline Enterococcus faecium & 7 & 15 & 0.01 \\
\hline Clostridium difficile & 5 & 8 & 0.032 \\
\hline Coagulase-negative Staphylococcus & 5 & 13 & 0.01 \\
\hline Methicillin-resistant Staphylococcus aureus & 4 & 8 & 0.02 \\
\hline Streptococcus agalactae & 4 & 2 & - \\
\hline Acinetobacter baumannii & 15 & 15 & - \\
\hline Enterococcus faecalis & 9 & 9 & - \\
\hline Burkholderia cephacia & - & 3 & - \\
\hline Staphylococcus epidermidis & 4 & 1 & - \\
\hline Morganella morganii & 3 & - & - \\
\hline Acinetobacter iwoffii & - & 2 & - \\
\hline Bacteroides eggerthi & - & 2 & - \\
\hline Serratia marcescens & - & 2 & - \\
\hline Streptococcus gallolyticus & - & 2 & - \\
\hline Trischosporon mucoides & - & 2 & - \\
\hline Micrococcus species & - & 1 & - \\
\hline Bacteroides thetaiotaomicron & - & 1 & - \\
\hline Citrobacter braakii & - & 1 & - \\
\hline Citrobacter freudii & - & 1 & - \\
\hline Streptococcus mitis & - & 1 & - \\
\hline Pseudomonas fluorescen & - & 1 & - \\
\hline Haemophilus influenzae & - & 1 & - \\
\hline Stenotrophomonas maltophilia & - & 1 & - \\
\hline Fusobacterium necrophorum & - & 1 & - \\
\hline Gemella morbillorum & - & 1 & - \\
\hline Candida albicans & 3 & 8 & - \\
\hline Enterobacter aerugenes & 3 & 2 & - \\
\hline Candida glabrata & 2 & 1 & - \\
\hline Bacillus specie & 2 & - & - \\
\hline Candida parapsilosis & 2 & - & - \\
\hline Streptococcus constellatus & 2 & - & - \\
\hline Providentia rettgeri & 2 & - & - \\
\hline Streptococcus anginosus & 1 & 1 & - \\
\hline Streptococcus haemolyticus & 1 & - & - \\
\hline Corynebacterium species & 1 & 1 & - \\
\hline Prevotella bivia & 1 & - & - \\
\hline Peptostreptococcus anaerobius & 1 & & - \\
\hline Clostridium perfringes & 1 & - & - \\
\hline Klebsiella oxytoca & 1 & 1 & - \\
\hline Micrococcus specie & 1 & - & - \\
\hline Trichosporon asashi & 1 & - & - \\
\hline Total & 160 & 208 & - \\
\hline
\end{tabular}

ASP, antibiotic stewardship programmes. 


\section{Appendix 3}

TABLE 1-A3: Consumption of most frequently used agents.

\begin{tabular}{|c|c|c|c|}
\hline \multirow[t]{2}{*}{ Most used antibiotics } & \multicolumn{3}{|c|}{ Indication } \\
\hline & Prophylaxis $(95 \% \mathrm{Cl})$ & Empiric $(95 \% \mathrm{Cl})$ & Targeted $(95 \% \mathrm{Cl})$ \\
\hline \multicolumn{4}{|c|}{ DDD per 1000 patient days in the baseline stage } \\
\hline Amoxicillin/clavulanic acid & $28.14(21.66-34.63)$ & 170.69 (147.14-194.24) & $28.26(19.21-37.31)$ \\
\hline Piperacillin/tazobactam & - & $147.57(61.86-233.29)$ & $22.91(15.07-30.74)$ \\
\hline Fluconazole & 52.08 & $83.14(49.14-117.15)$ & - \\
\hline Metronidazole & 0.15 & $19.46(7.35-31.57)$ & $3.87(1.73-6.00)$ \\
\hline Cefazolin & $7.99(6.75-9.24)$ & - & - \\
\hline \multicolumn{4}{|c|}{ DDD per 1000 patient days in the ASP stage } \\
\hline Amoxicillin/clavulanic acid & $12.41(8.45-16.38)$ & 107.05 (89.76-124.33) & $14.05(7.12-20.98)$ \\
\hline Piperacillin/tazobactam & - & $90.16(79.30-100.93)$ & $27.68(21.54-33.83)$ \\
\hline Fluconazole & $12.13(9.32-14.93)$ & $79.53(47.52-111.54)$ & $23.54(2.49-44.58)$ \\
\hline Ertapenem & - & $5.35(3.58-7.11)$ & $21.04(13.97-28.11)$ \\
\hline Cefazolin & 11.06 (6.25-15.87) & - & - \\
\hline
\end{tabular}

ASP, antibiotic stewardship programmes; $\mathrm{Cl}$, confidence interval, DDD, defined daily doses. 